Original article

Running Title: AAV gene transfer of factor IX fusion proteins

\title{
"Potential limits of AAV-based gene therapy with the use of new transgenes expressing Factor IX fusion proteins"
}

LE QUELLEC Sandra*\#£, DANE Allison*\$, ENJOLRAS Nathalie ${ }^{\# £, ~ M C ~ I N T O S H ~}$

Jenny $^{\$}$, ROSALES Cecilia $\$$, NEGRIER Claude ${ }^{\# £, ~ N A T H W A N I ~ A m i t ~}{ }^{\$}$.

*These authors participated equally to this original work

\#EA 4609-Hémostase et Cancer-Université Claude Bernard Lyon I - France

$£$ Laboratoire d'hématologie - Hospices Civils de Lyon

${ }^{\$}$ Research department of haematology - University of London Cancer Institute - United Kingdom

Corresponding author:

LE QUELLEC, Sandra

Unité d'hémostase Clinique - Hôpital Cardiologique Louis Pradel

59, Boulevard Pinel

69677 BRON, FRANCE

Tel : +33(0) 472118830

Fax : +33(0) 472118817

Email : sandra.le-quellec@ @chu-lyon.fr

Key words: adeno-associated virus, gene therapy, haemophilia, factor IX, albumin fusion, Fc fusion

Word count: 3,345 



\section{$\underline{\text { Abstract }}$}

Introduction: The variety of treatment for Haemophilia B (HB) has recently improved with the emergence of both AAV-based gene therapy and bioengineered human factor IX (hFIX) molecules with prolonged half-life due to fusion to either albumin (Alb) or immunoglobulin $\mathrm{Fc}$ fragment $(\mathrm{Fc})$.

Aim: Adeno-associated viral vectors (AAV) mediating expression of hFIX-Alb and hFIX-Fc fusion proteins was investigated for gene therapy of $\mathrm{HB}$ to explore if their extended half-life translates to higher plasma levels of FIX.

Methods: Single-stranded cross-packaged AAV2/8 vectors expressing hFIX-Alb, hFIXFc and hFIX were evaluated in-vitro, and in mice.

Results: Both hFIX-Alb and hFIX-Fc fusion proteins were synthesised and expressed as single chains of expected size following AAV mediated gene transfer in-vitro and invivo. The procoagulant properties of these hFIX-fusion proteins were comparable to wild-type hFIX. However, their expression levels were 3-fold lower than wild-type hFIX in-vivo most likely due to inefficient secretion.

Conclusion: This, the first, evaluation of hFIX-fusion proteins in the context of AAV gene transfer suggests that the hFIX-fusion proteins are secreted inefficiently from the liver, thus preventing their optimal use in gene therapy approaches. 


\section{$\underline{\text { Introduction }}$}

Two major advances have emerged recently for the treatment of haemophilia B (HB), an X-linked recessive bleeding disorder. The first is the development of extended halflife FIX molecules such as the human FIX-albumin (hFIX-Alb) and hFIXimmunoglobulin-Fc (hFIX-Fc) fusion proteins $[1,2]$. These engineered proteins have enabled patients to reduce the frequency of prophylaxis without compromising clinical outcomes $[3,4]$. The presence of the Fc domain or albumin allows interaction of the fusion protein with the neonatal $F c$ receptor $(F c R n)[2,5]$. Interaction of fusion proteins with FcRn delays protein degradation in the lysosome and facilitates recycling back into the circulation. The second development is gene therapy, which leads to stable, longterm, dose dependent, expression of FIX at between 1-6\% in patients with severe HB following a single administration of adeno-associated viral (AAV) vector encoding the wild-type hFIX gene [6]. This level of expression has altered the phenotype of the patients from severe to moderate or mild. Natural history studies show that patients with mild haemophilia who typically have FIX levels of 5-40\% of normal have a much lower risk of spontaneous bleeding. However, epidemiology studies indicate that haemophilia patients with baseline factor level of $>15 \%$ almost never have spontaneous haemorrhage [7]. Therefore, the ultimate goal of gene therapy should be to achieve stable expression at $\geq 15 \%$. With the current technology, this will require higher dose of AAV vectors, which will be associated with increased liver toxicity $[6,8]$. To address the challenge of mediating therapeutic expression of FIX using lower, potentially safer, vector doses, studies have employed a transgene expressing the hyperactive hFIX Padua variant [9], and recently showed clinical benefit [10]. However, the risk of such approach is the potential immunogenicity to the amino acid change to create hyperactive variant. 
In this study, we investigated if liver-targeted gene transfer with AAV vectors encoding the hFIX-Fc and hFIX-Alb proteins would lead to higher steady-state plasma FIX levels than possible when using the wild-type FIX. Our study shows that hFIX-Alb and hFIXFc fusion proteins with procoagulant activity are synthesised following AAV mediated gene transfer in-vitro in human liver cells lines and in-vivo in murine liver. Expression of these fusion proteins is, however, lower than that of wild type FIX. The mechanisms limiting expression of the fusion proteins were explored to define the underlying obstacles. 


\section{Material and methods}

\section{Construction and production of viral vectors}

We designed a transgene expressing human FIX (hFIX) fused to mature albumin cDNA through FIXa- or FVIIa-tissue factor (TF) complex-cleavable linker previously described [1] under the control of our previously described small liver specific LP1 promoter [11]. A pUC57 cloning vector containing 201 bp of the 3' end of humanly codon-optimised hFIX (hFIXco) cDNA sequence followed by full linker and mature albumin cDNA sequences, flanked by EcoNI and NheI restriction sites were synthesized (GenScript Biotech Corp., Nanjing, China) and cloned using the Quick ligation ${ }^{\mathrm{TM}}$ kit (New England Biolabs.Inc, Hitchin, United-Kingdom). The control pAV-LP1-hFIXcoSTOP-(Alb) vector expressing wild-type hFIX (hFIX-ctrl1) was created by introducing a single nucleotide substitution using Q $5{ }^{\circledR}$ site-directed mutagenesis kit (New England Biolabs.Inc) with specific primers, thus inducing a stop codon within the cleavable linker. To obtain pAV-LP1-hFIXco-Fc vector expressing hFIX covalently fused to the Fe fragment of human IgG1 (hinge, $\mathrm{CH} 2$, and $\mathrm{CH} 3$ domains) [2], the hFIX coding region was PCR amplified using Phusion ${ }^{\circledR}$ High fidelity polymerase (New England Biolabs.Inc) from our pAV-LP1-hFIXco with specific primers introducing an EcoRI restriction sites at the 5 '-end of the hFIX cDNA, and allowing the removal of the hFIX stop codon and the introduction of BamHI restriction site. The Fc region of human IgG1 (hinge, $\mathrm{CH} 2, \mathrm{CH} 3$ ) was extracted from an in-house plasmid using BamHI and MluI. Using 3-way ligation the hFIXco (no stop codon) and Fc fragments were directionally cloned into our pAV-LP1-hFIXco to form pAV-LP1-hFIXco-Fc. The control vector pAV-LP1-hFIXco-STOP-(Fc) expressing wild-type hFIX (hFIX-ctrl2) was created by inserting the Fc fragment into pAV-LP1-hFIXco after the stop codon of hFIX using 
restriction enzymes digestion with BamHI and MluI followed by ligation using Quick ligation $\mathrm{kit}^{\circledR}$. All four cDNA sequences were sequenced.

Single-stranded cross-packaged AAV2/8 (ssAAV2/8) expressing hFIX fusion transgenes were made by the triple transient transfection of subclonfluent human embryonic kidney (293T) cells method described before [12-14]. Recombinant ssAAV2/8 vectors (ssAAV2/8-LP1-hFIXco-Alb, ssAAV2/8-LP1-hFIXco-STOP-(Alb), ssAAV2/8-LP1-hFIXco-Fc, and ssAAV2/8-LP1-hFIXco-STOP-(Fc) viruses) were isolated with affinity column chromatography as described previously [15]. The genome titres of rAAV-hFIX vectors were determined by mean of a validated quantitative polymerase-chain-reaction (QPCR) assay [16].

\section{In-vitro assay}

Huh-7 cells (ECACC 01042712) were maintained in conditioned media containing 5 $\mu \mathrm{g} / \mathrm{ml}$ of vitamin $\mathrm{K}$ as described previously [17] and were transiently transfected with $1 \mu \mathrm{g}$ of pAV-LP1-hFIXco-Alb, pAV-LP1-hFIXco-STOP-(Alb), pAV-LP1-hFIXco-Fc, and pAV-LP1-hFIXco-STOP-(Fc) vectors using Fugene ${ }^{\circledR} 6$ (Roche, Neuilly-sur-Seine, France). Huh-7 cells were also transduced in-vitro at an MOI of $10^{5} \mathrm{vg} / \mathrm{cell}$ of all four corresponding ssAAV2/8 vectors, as previously reported [18]. Twenty-four hour conditioned media were collected as previously described [19] and assayed for FIX:Ag and FIX:C levels, and SDS-PAGE followed by immunoblotting. The intra cellular secretory traffic of hFIX-ctrl1, rFIX-Alb, rFIXFc proteins was analysed using various protease inhibitors and inhibitor of cellular protein transport diluted in $0.02 \%$ ethanol as described elsewhere [19]. Results were expressed as a percentage of values obtained with control culture medium containing $0.02 \%$ ethanol 


\section{Mice, tail-vein injection and blood collection}

All animal work was carried out in the United Kingdom in accordance with the standards set in the care and use of laboratory animals by the National Institutes of Health. Vector was injected into the tail vein of 7 - to 10 -weeks old wild-type male C57BL/J6 immunocompetent mice (Charles River, Oxford, UK) and immunodeficient

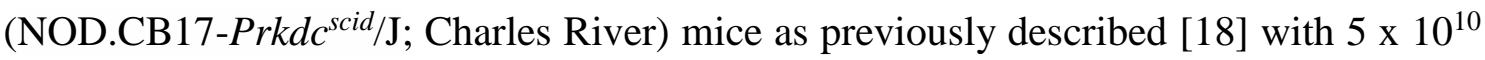
vg per mouse of respective rAAV vectors. Blood samples were collected following tailvein puncture regularly up to eight weeks after infusion. At termination, blood was collected from intra-cardiac puncture into $1 / 9$ volume $3.2 \%$ citrate microtubes. Mouse platelet-poor-plasma (PPP) was immediately prepared for the measurement of FIX:Ag level.

\section{Expression of hFIX constructs in mouse liver cells}

Experimental mouse livers were harvested at the time of sacrifice for genomic DNA isolation using DNeasy Blood and Tissue Kit (Qiagen, Valencia, CA, USA). Vector genome copy number in mouse liver was determined by QPCR as described previously [11]. To determine the level of mRNA transcripts, total RNA was extracted from mouse livers using RNeasy Blood \& Tissue kit (Qiagen). RNA analysis was performed using reverse transcription (SuperScript ${ }^{\mathrm{TM}}$ IV VILO ${ }^{\mathrm{TM}}$ Master Mix, Invitrogen) followed QPCR on liver tissue samples. Integrity of the DNA and the RNA were determined by amplifying a 295-bp region of the murine GAPDH gene.

For analysis of hFIX.Fc in mouse liver lysates, $\sim 0.05 \mathrm{~g}$ of liver was homogenised in lysis buffer $-0.5 \%$ v/v Triton X-100, 0.01M Hepes, $1 \mathrm{X}$ cOmplete ${ }^{\mathrm{TM}}$ Protease Inhibitor Cocktail (Roche Diagnostics, Meylan, France) - , clarified by centrifugation before 
protein concentration in lysate. Total proteins were quantified using Pierce ${ }^{\mathrm{TM}} \mathrm{BCA}$

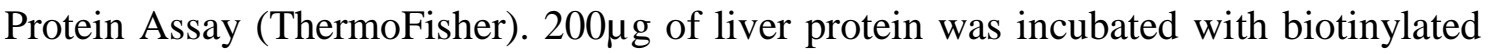
hFIX antibody (Affinity Biologicals) for 2 hours at $37^{\circ} \mathrm{C}$. Positive control of coagulation reference plasma (Technoclone, Vienna, Austria) was treated in the same way. Streptavidin-coupled Dynabeads (ThermoFisher) were washed with PBS$0.05 \%$ Tween buffer before the liver lysate-antibody mix was incubated with beads for 30 min with rotation. Bead mix was washed 3 times in PBS- $0.05 \%$ Tween before adding $10 \mu \mathrm{L}$ of reducing loading dye $(2.5 \mu \mathrm{L} 4 \mathrm{X}$ NuPAGETM LDS sample buffer (ThermoFisher) $6.5 \mu \mathrm{L}$ of water, $1 \mu \mathrm{L}$ of reducing agent), and was assessed for Western blot as described below.

\section{Factor IX detection and measurement}

FIX:Ag levels were determined using either human FIX specific ELISA as described previously $[18,20]$, or the ELISA ASSERACHROM:IX ${ }^{\circledR}$ Ag kit (Diagnostica Stago, Asnières, France). FIX:C levels were measured using the BIOPHEN chromogenic assay kit (Hyphen BioMed, Neuville-sur-Oise, France). Huh-7 cells and murine liver cell lysates samples were loaded onto a NuPAGETM 4-12\% Bis-Tris Protein Gel (Invitrogen ${ }^{\mathrm{TM}}$, Carlsbad, CA, USA) for electrophoresis under reducing conditions (using Tris 25mM, glycine $190 \mathrm{mM}$, SDS 4mM, pH 8.3 running buffer) and then transferred onto a nitrocellulose membrane (Amersham $^{\mathrm{TM}}$ Hybond ${ }^{\mathrm{TM}}-\mathrm{Cel}$, GEHealthcare, Saclay, France). The nitrocellulose membrane was then blocked with 5\% skimmed milk-PBS-0.05\% Tween $20^{\circledR}$ and exposed to HRP-conjugated affinity-purified goat anti-human FIX (1mg/ml) IgG (1:2000 dilution; Affinity biology, Ancaster, Ontario, Canada). Plasma samples from the mice were screened for the presence of $\mathrm{Ab}$ against hFIX using an ELISA method described previously [20]. 


\section{Statistical analysis}

Significant differences among groups were analysed via the non-parametric MannWhitney test Analysis was performed in GraphPad Prism (GraphPad Software, La Jolla, CA, USA). Results are expressed as mean \pm standard error to mean (SEM). A $P$ value of $<0.05$ was considered statistically significant. 


\section{$\underline{\text { Results }}$}

\section{rAAV vector genomes direct low expression of hFIX-fusion proteins in-vitro}

The albumin and human Fc fragments were cloned in-frame and downstream of the codon-optimised hFIX (hFIXco) gene, under the control of a liver-specific promoter to generate pAV-LP1-hFIXco-Alb (hFIX-Alb) and pAV-LP1-hFIXco-Fc (hFIX-Fc) AAV expression vectors, respectively. The Fc and albumin fusion sequences were downstream of the hFIX cDNA as described for the recombinant fusion proteins Alprolix $^{\circledR}$ and Idelvion ${ }^{\circledR}$. Control vectors were identical to these AAV expression cassettes with the exception of a stop codon introduced at the end of the FIX coding sequence to generate pAV-LP1-hFIXco-STOP-Alb (hFIX-ctrl1) pAV-LP1-hFIXcoSTOP-Fc (hFIX-ctrl2) vectors (Fig. 1A). Equivalent amounts $(1 \mu \mathrm{g})$ of all four pAV constructs were transiently transfected into Huh-7 cells, to biochemically characterise the fusion proteins and assess procoagulant activity. Conditioned media collected after 24 hours was assayed for protein expression by hFIX specific Western blot analysis and ELISA. Under reducing conditions, the apparent molecular weight of hFIX-Alb, hFIXFc and hFIX-ctl1/2 were approximately $140 \mathrm{kDa}, 100 \mathrm{kDa}$ and $60 \mathrm{kDa}$, respectively (Fig. 1B). The FIX mean antigen levels ( \pm SEM) for hFIX-Alb $1.33 \%( \pm 0.07)$ and hFIX-Fc $1.14 \%( \pm 0.04)$ were 7 to 8 -fold lower than hFIX-ctrl1 $9.18 \%( \pm 0.48)$; $\mathrm{p}<0.0001)$. The hFIX-Alb recombinant protein, Idelvion ${ }^{\circledR}$, was used as control in the same ELISA, and was detected at the expected antigen value, indicating that the lower FIX:Ag levels for the hFIX-fusion proteins on ELISA were not due to changes in conformation of the engineered proteins affecting detection [21]. The activity to antigen 
ratio of the fusion proteins was $\sim 1: 1$ suggesting that the fusion proteins were fully functional (Fig. 1C).

Next we produced and purified single-stranded AAV8 capsid pseudotyped vectors (ssAAV2/8) expressing the hFIX fusion proteins. The vector yields for the four vectors ranged from $1.74 \times 10^{12} \mathrm{vg} / \mathrm{ml} \pm 2.18 \times 10^{11}$ with no significant differences in titres between vectors encoding hFIX-Alb and hFIX-Fc and their respective controls. In-vitro transduction of Huh-7 cells resulted in expression of hFIX-Alb, hFIX-Fc as single chain peptides as described above (data not shown).

\section{Evaluation of AAV vectors encoding hFIX-fusion proteins in-vivo}

To evaluate the hFIX fusion proteins in-vivo, adult male C57BL/6 mice were injected with $5 \times 10^{10}$ vg per mouse of respective ssAAV2/8 vectors via the tail vein. Blood samples were collected at regular intervals over a period of eight weeks after gene transfer and assayed for hFIX:Ag levels. The kinetics of expression was similar for all four constructs with expression reaching steady state levels between 4-6 weeks after gene transfer. As with the in-vitro studies, both hFIX-Alb and hFIX-Fc proteins were detected at significantly lower levels than hFIX-ctrl1/2, with mean $( \pm$ SEM) steady state FIX:Ag levels of $38.86 \%( \pm 1.30)$ for hFIX-Alb and $227.40 \%$ (14.57) for hFIX-ctrl1 $(\mathrm{p}<0.0001)$, and $60.30 \%( \pm 14.04)$ for hFIX-Fc and $167.90 \%( \pm 17.08)$ for hFIX-ctrl2 ( $\mathrm{p}=0.0006$; Fig.2A). No significant difference were seen between hFIX-Alb and hFIXFc levels of expression $(\mathrm{p}=0.2773)$. Antibodies to hFIX were not detected (data not shown) in any of the cohorts, thus ruling out a neutralising antibody response to the FIX-fusion proteins as a potential explanation for low plasma hFIX levels. Consistent with this the mean steady-state FIX:Ag level of the hFIX-Fc $(24.45 \% \pm 4.70)$ was 
significantly lower than hFIX-ctrl2 $(72.68 \% \pm 18.05 ; \mathrm{p}=0.0286)$ following AAV mediated gene transfer of the respective constructs in immunodeficient mice.

Vector genome copy number in mouse liver did not show a significant difference in proviral DNA in the hFIX-Fc cohort when compared to hFIX-ctrl2 $(13.9 \pm 5.2 \mathrm{vg} / \mathrm{cell}$ and $24 \pm 2.7 \mathrm{vg} /$ cell, respectively; $\mathrm{p}=0.100 ;$ Fig. $2 \mathrm{~B}$ ). In contrast, the copy number for hFIX-Alb proviral DNA in the livers of hFIX-Alb cohort was significantly higher when compared to hFIX-ctrl1 (61.7 \pm 7.9 vg/cell vs $16 \pm 5.7$ vg/cell; $p=0.0286$; Fig. $2 \mathrm{~B})$. This most likely reflected an underestimation of the hFIX-Alb vector titre, leading to administration of a higher vector dose than planned.

To determine if the reduced expression from AAV2/8-hFIX-Alb or -hFIX-Fc was due to inefficient transcription, we performed RNA analysis on liver tissue samples. The mRNA levels present in the hFIX-Alb cohort were lower compared to control samples, potentially suggesting altered transcription of the hFIX-Alb cDNA. There was a trend toward higher mRNA levels in the hFIX-Fc cohort compared to the control samples (Fig. 2C). This may indicate inefficient translation, protein misfolding and degradation, increased mRNA stability or inefficient secretion of the fusion construct.

\section{Low expression of hFIX fusion proteins following gene transfer may be due to poor secretion from murine hepatocytes}

Limited immunoprecipitation studies of murine liver cell-lysates showed that hFIX-Fc protein was present at relatively high levels within the hepatocytes when compared to controls (Fig. 3A). This result suggested an alteration of the hFIX-Fc fusion protein secretion. To further understand this observation, we next assessed celltrafficking of hFIX variants in Huh-7 cells following transfection with plasmids 
encoding hFIX-Alb and hFIX-Fc and hFIX-ctrl1/2 to understand the reasons for poor secretion of the hFIX-fusion proteins. Intracellular FIX concentrations were measured by ELISA. The secretion profiles of hFIX-Alb and hFIX-ctrl1 were similar, showing an increase in intracellular accumulation of hFIX-Alb and hFIX-ctrl1 following blockade of endoplasmic reticulum to Golgi transit by brefeldin-A but no change in intracellular FIX levels with either $\beta$-lactone, a proteasome inhibitor, or $\mathrm{NH}_{4} \mathrm{Cl}$ an inhibitor of lysosomal activity. The trafficking profile for hFIX-Fc showed a degree of variation with a significant reduction of hFIX-Fc after inhibition of lysosomal activity (Fig. 3B). 


\section{$\underline{\text { Discussion }}$}

The treatment of $\mathrm{HB}$ is undergoing substantial changes, with the approval of extended half-life clotting factor concentrates and the parallel, ongoing validation of FIX gene therapy in several clinical trials. Covalent fusion of the Fc domain of human immunoglobulin, IgG1 or human albumin with recombinant hFIX protects the protein from intracellular lysosomal degradation and facilitates its recycling back into the circulation. This results in a 3-5 fold increase in the plasma half-life of FIX, which enables the reduction in the frequency of factor infusion in patients with severe HB. These products raise the possibility of consistently achieving higher trough levels leading to prevention of breakthrough bleeding and subsequent joint disease. Furthermore, animal studies have not suggested an increased inhibitor rate with extended half-life products $[1,2,22]$. Therefore, these hFIX-fusion proteins are highly attractive for the next generation of gene therapy vectors, primarily because of the possibility of increasing steady-state plasma FIX levels due to their extended half-life, thus improving the potency of gene transfer without having to increase the vector dose and associated toxicity $[6,8]$.

In the knowledge that $\mathrm{hFIX}-\mathrm{Fc}$ and hFIX-Alb recombinant proteins were amenable to production at scale, we set out to evaluate these long-acting hFIX-fusion proteins in the context of AAV gene therapy. Whilst these fusion proteins, when expressed following gene transfer, had the expected procoagulant activity with an antigen:activity ratio of 1:1, their level of expression was significantly lower levels than wild-type hFIX following AAV-mediated gene transfer into the liver of mice or following transient transfection of the AAV expression cassette plasmid into human liver cell lines. The observed reduced expression from both plasmid and AAV vectors 
suggests a potentially cross-platform issue with expression from liver cells that is likely to be observed with other gene transfer vectors including integrating vectors such as those based on lentivirus.

The mechanism behind the reduced plasma expression of hFIX-Fc and hFIXAlb following gene transfer observed in this study remains unclear. Preliminary analysis suggests that the reasons for the poor expression of the two hFIX-fusion proteins may be different. Notably, the mRNA levels present in the hFIX-Alb cohort were not different compared to control samples despite higher in-vivo transduction rate. This suggests inefficient transcription of the hFIX-Alb proviral vector. The trend for higher mRNA levels after hFIX-Fc delivery compared to controls may indicate increased mRNA stability or a subsequent issue with protein secretion. Numerous examples in the literature exist of increased mRNA stability mediated by 3' regions of genes including the globin genes [23]. The possibility that improved mRNA stability mediated by the Fc moiety, which is downstream of the hFIX cDNA, exists. The limited immunoprecipitation studies of murine liver cell-lysates showed that hFIX-Fc protein was present at relatively high levels within the hepatocytes when compared to controls, suggesting an alteration of the hFIX-Fc fusion protein secretion. In addition, intracellular trafficking studies indicated that the secretion profile of hFIX-Fc fusion proteins was different than the ones of hFIX-Alb and hFIX-ctrl1. Studies with $\mathrm{NH}_{4} \mathrm{Cl}$, which inhibits the autophagic flux into lysosomes, led to hFIX-Fc proteins be redirected from the lysosomal compartment to organelles such as endosome with a quicker degradation rate [24], or increased the level of misfolded hFIX-Fc protein within the cell resulting in elevated endoplasmic-reticulum stress and a higher apoptotic rate [25]. These data suggest that the autophagy-lysosome pathway, involved in the intracellular 
protein quality control [26] by removing large misfolded proteins and proteins aggregates [27], is activated in liver cells expressing hFIX-Fc fusion proteins [28]. This hypothesis is in accordance with the propensity of Fc fusion proteins to form protein aggregates [28], which is mostly induced by hydrophobic interactions [29]. Indeed, it has been shown that the coupling of the Fc region to the hFIX resulted in a difference in hydrogen atom exposition of residues 408-414 [30], which potentially favoured hydrophobic interactions and subsequent formation of hFIX-Fc fusion protein aggregates.

Our hypothesis, therefore, is that the lower plasma circulating levels of the hFIX-fusion proteins is likely due to inefficient secretion of these proteins from hepatocytes when compared to wild-type hFIX protein. There are reports of mutations within FIX or factor VIII (FVIII) genes leading to changes in protein configuration and poor secretion from the producer cells $[19,31]$. The role of PACE/furin expression could also be considered as a potential limiting underlying mechanism for low hFIX fusion protein secretion [32, 33]. It is intriguing to speculate whether similar observations are made during the large-scale manufacture of these proteins as recombinant concentrates, though it is possible that changes in secretion pattern of hFIX-fusion protein may not be detected if the producer cells are lysed prior to purification of the hFIX-fusion protein.

One limitation of our study is that we did not inject HB mice, which would have allowed evaluating the hFIX activity level and the resulting correction of the bleeding phenotype after gene transfer of AAV vectors expressing hFIX-fusion proteins. However, both hFIX-fusion proteins have a lower specific activity compared to wildtype hFIX $[1,2]$. This suggested that the lower hFIX:Ag level for both hFIX-fusion 
proteins may have translated to an even lower hFIX activity level and a less efficient correction of the bleeding phenotype compared to hFIX-ctrl.

In light of these results we believe the way forward for improving the efficiency of AAV mediated gene transfer is to use a gain-of-function, hyperactive form of FIX. Indeed results from a recent phase I/II clinical trial [10] showed that the use of the hFIX-Padua variant, a naturally occurring variant with approximately 8-fold higher specific activity compared to wild-type hFIX [9], in an AAV vector, could result in sustained mean steady-state FIX:C of about 34\%. Interestingly, the FIX:Ag expression levels, in patients who had a negative status for cross-reactive material, were similar to that observed in the previous clinical trial sponsored by UCL/St Jude $[6,10]$.

\section{$\underline{\text { Conclusion }}$}

In conclusion, our study shows that hFIX-fusion proteins, though synthesized by hepatocytes appropriately, are not efficiently secreted, thus making them unsuitable for use in gene therapy approaches, despite their extended half-life as recombinant protein replacement therapies. Our preliminary data suggests that the intracellular trafficking of hFIX-Alb and hFIX-Fc may differ but further studies are required to elicit the mechanism behind these observations. 


\section{$\underline{\text { Authorship contributions }}$}

S Le Quellec and A Dane designed the study, performed experiments and wrote the manuscript. C Rosales performed animal experiments. J Mc Intosh, N Enjolras, C Négrier, and A Nathwani critically revised the manuscript and gave final approval.

\section{$\underline{\text { Acknowledgement }}$}

Idelvion ${ }^{\circledR}$ was kindly provided by CSL Behring. This work was supported by a grant from the "Fondation Groupama pour la Santé - Vaincre les maladies rares" for financial support. We also thank Jonathan Girard for his technical skills and expertise. A Dane is currently an employee of Freeline Therapeutics. C Négrier has acted as a paid consultant for Freeline Therapeutics. A Nathwani is an advisor to Freeline, Biomarin, and GenerationBio, he is a founder of and has a research agreement with Freeline Therapeutics, he holds equity in Freeline Therapeutics, he is a consultant to several biopharmaceutical companies and he is an inventor on patents licensed to Freeline Therapeutics and Biomarin. S Le Quellec, N Enjolras, C Rosales, J McIntosh, and Y Dargaud have no competing interests. 


\section{$\underline{\text { References }}$}

1. Metzner HJ, Weimer T, Kronthaler U, Lang W, Schulte S. Genetic fusion to albumin improves the pharmacokinetic properties of factor IX. Thromb Haemost 2009; 102: 634-44.

2. Peters RT, Low SC, Kamphaus GD, Dumont JA, Amari JV, Lu Q, et al. Prolonged activity of factor IX as a monomeric Fc fusion protein. Blood 2010; 115: 2057-64.

3. Santagostino E, Martinowitz U, Lissitchkov T, Pan-Petesch B, Hanabusa H, Oldenburg J, et al. Long-acting recombinant coagulation factor IX albumin fusion protein (rIX-FP) in hemophilia B: results of a phase 3 trial. Blood 2016; 127: 1761-9.

4. Powell JS, Pasi KJ, Ragni MV, Ozelo MC, Valentino LA, Mahlangu JN, et al. Phase 3 study of recombinant factor IX Fc fusion protein in hemophilia B. N Engl J Med 2013; 369: 2313-23.

5. Chaudhury C, Mehnaz S, Robinson JM, Hayton WL, Pearl DK, Roopenian DC, et al. The major histocompatibility complex-related Fc receptor for IgG (FcRn) binds albumin and prolongs its lifespan. J Exp Med 2003; 197: 315-22.

6. Nathwani AC, Tuddenham EG, Rangarajan S, Rosales C, McIntosh J, Linch DC, et al. Adenovirus-associated virus vector-mediated gene transfer in hemophilia B. N Engl J Med 2011; 365: 2357-65.

7. den Uijl IE, Fischer K, Van Der Bom JG, Grobbee DE, Rosendaal FR, Plug I. Analysis of low frequency bleeding data: the association of joint bleeds according to baseline FVIII activity levels. Haemophilia 2011; 17: 41-4.

8. Nathwani AC, Reiss UM, Tuddenham EG, Rosales C, Chowdary P, McIntosh J, et al. Long-term safety and efficacy of factor IX gene therapy in hemophilia B. N Eng/ J Med 2014; 371: 1994-2004.

9. Simioni P, Tormene D, Tognin G, Gavasso S, Bulato C, lacobelli NP, et al. X-linked thrombophilia with a mutant factor IX (factor IX Padua). N Eng/ J Med 2009; 361: 1671-5. 10. George LA, Sullivan SK, Giermasz A, Rasko JEJ, Samelson-Jones BJ, Ducore J, et al. Hemophilia B Gene Therapy with a High-Specific-Activity Factor IX Variant. N Engl J Med 2017; 377: 2215-27.

11. Nathwani AC, Gray JT, Ng CY, Zhou J, Spence Y, Waddington SN, et al. Selfcomplementary adeno-associated virus vectors containing a novel liver-specific human factor IX expression cassette enable highly efficient transduction of murine and nonhuman primate liver. Blood 2006; 107: 2653-61.

12. Nathwani AC, Hanawa $H$, Vandergriff J, Kelly P, Vanin EF, Nienhuis AW. Efficient gene transfer into human cord blood CD34+ cells and the CD34+CD38- subset using highly purified recombinant adeno-associated viral vector preparations that are free of helper virus and wildtype AAV. Gene Ther 2000; 7: 183-95.

13. Allay JA, Sleep S, Long S, Tillman DM, Clark R, Carney G, et al. Good manufacturing practice production of self-complementary serotype 8 adeno-associated viral vector for a hemophilia B clinical trial. Hum Gene Ther 2011; 22: 595-604.

14. Davidoff AM, Ng CY, Sleep S, Gray J, Azam S, Zhao Y, et al. Purification of recombinant adeno-associated virus type 8 vectors by ion exchange chromatography generates clinical grade vector stock. J Virol Methods 2004; 121: 209-15.

15. Clark WM, Kassick AJ, Plotkin MA, Eldridge AM, Lantos I. A highly enantioselective conjugate reduction of 3-arylinden-1-ones using bakers' yeast for the preparation of (S)-3arylindan-1-ones. Org Lett 1999; 1: 1839-42.

16. Fagone P, Wright JF, Nathwani AC, Nienhuis AW, Davidoff AM, Gray JT. Systemic errors in quantitative polymerase chain reaction titration of self-complementary adeno-associated viral vectors and improved alternative methods. Hum Gene Ther Methods 2012; 23: 1-7. 
17. Enjolras N, Perot E, Le Quellec S, Indalecio A, Girard J, Negrier C, et al. In vivo efficacy of human recombinant factor IX produced by the human hepatoma cell line HuH-7.

Haemophilia 2015; 21: e317-21.

18. Nathwani AC, Davidoff A, Hanawa H, Zhou JF, Vanin EF, Nienhuis AW. Factors influencing in vivo transduction by recombinant adeno-associated viral vectors expressing the human factor IX cDNA. Blood 2001; 97: 1258-65.

19. Enjolras N, Plantier JL, Rodriguez $\mathrm{MH}$, Rea $\mathrm{M}$, Attali O, Vinciguerra $\mathrm{C}$, et al. Two novel mutations in EGF-like domains of human factor IX dramatically impair intracellular processing and secretion. J Thromb Haemost 2004; 2: 1143-54.

20. Nathwani AC, Davidoff AM, Hanawa H, Hu Y, Hoffer FA, Nikanorov A, et al. Sustained high-level expression of human factor IX (hFIX) after liver-targeted delivery of recombinant adeno-associated virus encoding the hFIX gene in rhesus macaques. Blood 2002; 100: 1662-9. 21. Martinowitz U, Lubetsky A. Phase I/II, open-label, multicenter, safety, efficacy and PK study of a recombinant coagulation factor IX albumin fusion protein (rIX-FP) in subjects with hemophilia B. Thromb Res 2013; 131 Suppl 2: S11-4.

22. Nolte MW, Nichols TC, Mueller-Cohrs J, Merricks EP, Pragst I, Zollner S, et al. Improved kinetics of rIX-FP, a recombinant fusion protein linking factor IX with albumin, in cynomolgus monkeys and hemophilia B dogs. J Thromb Haemost 2012; 10: 1591-9.

23. Clegg JB, Weatherall DJ, Milner PF. Haemoglobin Constant Spring--a chain termination mutant? Nature 1971; 234: 337-40.

24. Hart PD, Young MR. Ammonium chloride, an inhibitor of phagosome-lysosome fusion in macrophages, concurrently induces phagosome-endosome fusion, and opens a novel pathway: studies of a pathogenic mycobacterium and a nonpathogenic yeast. J Exp Med 1991; 174: 881-9.

25. Kim I, Xu W, Reed JC. Cell death and endoplasmic reticulum stress: disease relevance and therapeutic opportunities. Nat Rev Drug Discov 2008; 7: 1013-30.

26. Dikic I. Proteasomal and Autophagic Degradation Systems. Annu Rev Biochem 2017;

86: 193-224.

27. Lamb CA, Yoshimori T, Tooze SA. The autophagosome: origins unknown, biogenesis complex. Nat Rev Mol Cell Biol 2013; 14: 759-74.

28. Huang RY, lacob RE, Krystek SR, Jin M, Wei H, Tao L, et al. Characterization of Aggregation Propensity of a Human Fc-Fusion Protein Therapeutic by Hydrogen/Deuterium Exchange Mass Spectrometry. J Am Soc Mass Spectrom 2017; 28: 795-802.

29. Mahler HC, Friess W, Grauschopf U, Kiese S. Protein aggregation: pathways, induction factors and analysis. J Pharm Sci 2009; 98: 2909-34.

30. Houde D, Berkowitz SA. Conformational comparability of factor IX-Fc fusion protein, factor IX, and purified Fc fragment in the absence and presence of calcium. J Pharm Sci 2012; 101: $1688-700$.

31. Pipe SW, Kaufman RJ. Factor VIII C2 domain missense mutations exhibit defective trafficking of biologically functional proteins. J Biol Chem 1996; 271: 25671-6.

32. Wasley LC, Rehemtulla A, Bristol JA, Kaufman RJ. PACE/furin can process the vitamin Kdependent pro-factor IX precursor within the secretory pathway. J Biol Chem 1993; 268: 845865.

33. Siner JI, Samelson-Jones BJ, Crudele JM, French RA, Lee BJ, Zhou S, et al. Circumventing furin enhances factor VIII biological activity and ameliorates bleeding phenotypes in hemophilia models. JCI Insight 2016; 1: e89371. 


\section{Figures caption list}

Fig.1 Schematic diagram of hFIX vector genomes and expression of hFIX molecules in-vitro. A) AAV2-based expression cassettes containing hFIX fused to human albumin or human IgG1 Fc, flanked by SV40 small $\mathrm{t}$ antigen intron before the start codon of hFIX and a SV40 polyA sequences, and under the transcriptional control of liver specific promoter LP1. Equivalent control vector genomes (ctrl1 and ctrl2) were prepared by inserting a stop codon between the FIX sequence and the fused protein, ensuring the albumin or Fc sequence was not translated. The albumin fusion constructs contained a FXIa/FVIIa-tissue factor complex- cleavable linker. ITR, inverted terminal repeat; bp, base pair; hFIXco, codon-optimised human factor IX; Alb, human albumin; Fc, Fc region of human IgG1 (hinge, $\mathrm{CH}_{2}, \mathrm{CH}_{3}$ ); ctrl1/2, control vector 1/2; B) AntihFIX Western blot on culture medium of transiently transfected Huh-7 cells with pAV expression vectors. The hFIX-Alb fusion construct and its control (hFIX-ctrl1) are shown in the left side of the figure 1B, and hFIX-Fc fusion construct and its control (hFIX-ctrl2) are shown in the right side of the figure 1B. NT=not transfected. C) FIX:Ag levels and FIX:C levels on culture medium of transiently transfected Huh-7 cells. Idelvion ${ }^{\circledR}$ spiked at $4 \%$ was used as control. NT $=$ not transfected. Mann-Whitney test was performed for comparison of hFIX-Alb or hFIX-Fc result and hFIX-ctrl1 groups.

Fig.2 Expression of hFIX fusion molecules in-vivo. A) Effect of Albumin and Fc fusion to FIX on level of FIX expression in-vivo. Wild-type male C57Bl/6 mice were injected with $5 \times 10^{10}$ vg per mouse of AAV2/8.hFIX-Alb (n=4), AAV2/8.hFIX-Fc (n=3) or AAV2/8.hFIX ctrl1/2 $(n=4 ; n=3)$ vectors. B) DNA was extracted from livers harvested from each treatment group and the number of vector genomes per diploid cell 
was determined in triplicate using QPCR. C) Relative hFIX mRNA copies per vg per cell from liver tissue were determined in triplicate by RT-QPCR. Mann-Whitney test was performed for comparison between hFIX-Alb and hFIX-ctrl1 groups, and between hFIX-Fc and hFIX-ctrl2 groups. Error bars represent mean \pm SEM.

Fig.3 hFIX fusion protein expression in mouse liver and in-vitro intracellular trafficking. A) hFIX immunoprecipitation (IP) of mouse liver lysates followed by Western with anti-hFIX antibody. Coagulation reference plasma was used as a positive control. B) Intracellular trafficking experiment. Mann-Whitney test was performed for comparison between FIX:Ag values obtained after incubation with inhibitors and those obtained from untreated Huh-7 cells for each vector group. Error bars represent SEM. Results were expressed as a percentage of values obtained with control culture medium containing $0.02 \%$ ethanol. 